\title{
Factores estresores y síndrome de Burnout en enfermeras de cuidados intensivos en dos hospitales de EsSalud nivel III-2 Lima Metropolitana
}

\author{
Maria del Rosario Menor-Segura, ${ }^{\mathrm{a}, \mathrm{b}}$ Rosa Mary Díaz-Sotelo, ${ }^{\mathrm{a}, \mathrm{b}}$ Luz Olinda Fernández-Henríquez. ${ }^{\mathrm{c}, \mathrm{d}}$
}

\begin{abstract}
Menor-Segura MR, Díaz-Sotelo RM, FernándezHenríquez LO. Factores estresores y síndrome de Burnout en enfermeras de cuidados intensivos en dos hospitales de EsSalud nivel III-2 Lima Metropolitana. Cuid salud, ene-jun 2015; 2(1):137147.
\end{abstract}

\section{RESUMEN}

Estudios de Europa determinan la prevalencia de Burnout en enfermeras entre 10-30\%, en Perú afecta al $11 \%$, con gran repercusión en la salud física y mental; siendo las unidades de cuidados intensivos un área de alta presión laboral y carga psico-emocional. Objetivo: determinar los factores estresores asociados al síndrome de Burnout en enfermeras de UCI de hospitales de EsSalud nivel III-2, Lima-Metropolitana. Metodología: estudio cuantitativo, diseño descriptivo correlacional, considerando una población de 114 enfermeras de UCI, se utilizó dos instrumentos: Inventario de Burnout de Maslach y cuestionado de Respuesta Emocional al Estrest laboral. Los datos se recolectaron en agosto/2014, fueron analizados en paquete estadístico SPSS v.21 usando estadística descriptiva para las univariables y $\mathrm{chi}^{2}$ de Pearson para las bivariables. Resultados: el 60\% de enfermeras presenta factores estresores en nivel medio y $22 \%$ nivel alto, destacándose en el análisis por factores "presión y exigencia" y "organizativos y relaciones humanas" con nivel medio y "ambientales" nivel alto. Respecto al síndrome de Burnout, el $7 \%$ estaba quemado y $84 \%$ tenía tendencia a desarrollarlo. La correlación de variables mostró que los factores estresores están asociados con el síndrome de Burnout. Conclusiones: existe relación entre las variables, siendo necesario implementar mejoras acorde con las recomendaciones internacionales.

Palabras clave: factores estresores, cuidado, enfermera, sobrecarga laboral.
Menor-Segura MR, Díaz-Sotelo RM, FernándezHenríquez LO. Stress factors and burnout syndrome in intensive care nurses among two hospitals in EsSalud Level III-2 Metropolitan Lima. Cuid salud, ene-jun 2015; 2(1):137-147.

\begin{abstract}
European Studies determine the prevalence of Burnout in nurses between 10-30\%, in Peru, affects $11 \%$, with consequences in physical and mental health; It is the intensive care units (ICU) an area of high work pressure and psychoemotional load. Objective: To Determine the stress factors associated to Burnout syndrome in ICU nurses of EsSalud hospital level III-2Metropolitan Lima. Methodology: Quantitative study, descriptive correlational design, considering a population of 114 ICU nurses, two instruments were used: Maslach Burnout Inventory and Emotional Response questioned work stress. Data were collected in August 2014, were analyzed using SPSS v.21 for univariate descriptive statistics and Pearson $\mathrm{chi}^{2}$ for bivariate. Results: $60 \%$ of nurses show stressors factors in middle level and $22 \%$ at high level, distinguishing in analysis factors "pressure and demand" and "organizational and human relations" with midlevel and "environmental" with high level. Regarding the burnout syndrome, $7 \%$ was burned and $84 \%$ had a tendency to develop it. The correlation of variables showed that stress factors are associated with burnout syndrome. Conclusions: There is relation between variables. It is necessary to implement improvements in line with international recommendations.
\end{abstract}

Keywords: Stress factors, nursing, nurse, work overload.

\footnotetext{
a Licenciada en Enfermería.

Estudiante de la Especialidad en Centro Quirúrgico, Escuela de Enfermería Padre Luis Tezza, afiliada a la Universidad Ricardo Palma, Lima-Perú.

Magister en Salud Pública.

Docente de la Escuela de Enfermería Padre Luis Tezza afiliada a la Universidad Ricardo Palma, Lima-Perú.
} 


\section{INTRODUCCIÓN}

A nivel mundial el estrés ocupacional crónico es un factor fundamental para desarrollar trastornos mórbidos y entre ellos la depresión, reconocido como la cuarta causa de enfermedad en el trabajador de salud. Según la Organización Mundial de la Salud (OMS), ${ }^{1,2}$ en el 2020 la depresión sería la segunda causa de morbilidad, aumentando las licencias por enfermedad, jubilación anticipada y abandono de la profesión. Anualmente, 160 millones de nuevos casos de enfermedad estarían relacionados con el trabajo.

En el Reino Unido, estudios sobre enfermedades relacionadas con el trabajo han identificado que, aproximadamente 91000 nuevos casos son ocasionados en su mayoría por el estrés. ${ }^{3}$ Un procedimiento de investigación sobre el origen de las enfermedades laborales mostró que el $64 \%$ de las patologías provenía de enfermedades profesionales declaradas y $36 \%$ era por sospecha de enfermedad laboral. ${ }^{4}$

La Organización Internacional del Trabajo $(\mathrm{OIT})^{5}$ refiere que las enfermedades profesionales y los accidentes laborales producen una pérdida de 2,8 billones de dólares anuales a nivel mundial, correspondiente al $4 \%$ del producto bruto interno (PBI); siendo los síntomas más habituales el cansancio físico y mental, cuyas posibles causas son la falta de éxito terapéutico observable en los pacientes, falsos liderazgos y falta de interacción social. ${ }^{6}$

Estudios realizados en Europa estiman la prevalencia del síndrome de Burnout en enfermeras entre el $10-30 \% .^{7-9} \mathrm{La}$ encuesta "Barómetro del estrés en enfermería" en España mostró que el $96 \%$ había experimentado en alguna ocasión estrés en el trabajo, ${ }^{7}$ con niveles bajos de realización personal. ${ }^{8}$ En Francia, las enfermeras de cuidados críticos reportaron niveles graves de Burnout $(32,8 \%)$ y $60 \%$ expresó que desearía cambiar de profesión. ${ }^{9}$

En América Latina y El Caribe existen también varios estudios. ${ }^{10-15}$ Argentina, Brasil y Costa Rica muestran mayor nivel de Burnout, problema que afecta al $43-55 \%$ de enfermeras y al $11 \%$ en el Perú. ${ }^{15}$ Un estudio realizado en la unidad de cuidado crítico del Hospital Central de la Fuerza Aérea del Perú reportó niveles altos de despersonalización (15\%); ${ }^{16}$ en Chiclayo, el $20 \%$ de enfermeras tenía niveles altos de cansancio emocional. ${ }^{17}$ En general, los peruanos presentan algún grado de estrés laboral según una encuesta realizada por el Comercio. ${ }^{18}$

Maslach $^{19}$ define al Burnout como un síndrome psicológico que se origina bajo ciertas experiencias interrelacionadas: primero aparece el agotamiento como respuesta al estrés, luego se produce un cambio negativo sobre cómo se siente uno con el trabajo y con las demás personas; posteriormente se manifiestan sentimientos de negatividad sobre sí mismo, su capacidad, competencias, deseos y motivaciones para trabajar. Según la $\mathrm{OMS},{ }^{2}$ la presencia de este síndrome provoca detrimento en la salud física y mental del trabajador.

Los profesionales de enfermería de áreas críticas son el personal más vulnerable al síndrome de Burnout, debido al esfuerzo físico y mental que realizan al cuidar a pacientes de alta complejidad, al mismo tiempo que deben dominar la tecnología de punta de los equipos del servicio -sin estar suficientemente capacitadas- adicionándose a esta situación el dolor y las necesidades de los familiares. Todos ellos constituyen factores estresores del contexto hospitalario, siendo posible diferenciar a los personales, la presión y la exigencia (de los superiores), la organización y las relaciones humanas (estructura organizacional y cultura) y los ambientales (infraestructura y equipos).

Los factores personales pueden ocasionar frustración cuando la enfermera no logra sus expectativas a nivel profesional; el factor presión y exigencia puede originar estrés cuando el profesional de supervisión es autoritario e imponente, infundiendo miedo en su personal subalterno; finalmente, el factor organizativo (del servicio) y de relaciones humanas no siempre son bien toleradas por las enfermeras, ya que no se adaptan totalmente a las normas que rige en la institución. $^{20}$

En la práctica, las enfermeras con escasa experiencia clínica y las enfermeras versadas de las unidades de cuidados intensivos (UCI), aunque trabajan de forma colaborativa, no siempre se desarrollan en consonancia. En ocasiones las primeras son sometidas a alta presión por sus colegas de mayor experiencia y eventualmente escarnecidas en público; dejando traslucir miedo e inseguridad en el desarrollo de técnicas y procedimientos, situación que les produce desasosiego para desenvolverse en sus máxima capacidad. 
Bajo este contexto y reconociendo que las condiciones laborales, personales y sociales de las enfermeras en UCI son estresantes, es necesario evaluar periódicamente la interacción de estos factores en la perspectiva de introducir cambios que ayuden al mejor desarrollo profesional $\mathrm{y}$ contribuyan a un cuidado de calidad en favor del paciente y de sí mismas. En ese sentido, el objetivo de este estudio fue determinar los factores estresores asociados al síndrome de Burnout en enfermeras de cuidados intensivos de hospitales de EsSalud nivel III-2, Lima Metropolitana, en el tercer trimestre 2014.

\section{METODOLOGÍA}

Estudio de enfoque cuantitativo, método observacional, de tipo aplicativo y diseño correlacional, de corte trasversal; realizado en dos hospitales de EsSalud nivel III-2 de Lima Metropolitana: Hospital Guillermo Almenara Irigoyen (A), ubicado en el distrito de La Victoria y el Hospital Edgardo Rebagliati Martins (B), en el distrito de Jesús María. Ambos hospitales brindan una atención especializada e integral, con servicios de salud de alta capacidad resolutiva tanto en el campo clínico como en los grupos etarios, desarrollando actividades de consulta externa, hospitalización y capacitación, investigación y docencia.

La población fue compuesta por enfermeras que laboran en unidades de cuidado intensivo (UCI) adultos, haciendo un total de 119 enfermeras $(\mathrm{A}=48$ y $\mathrm{B}=71)$, las mismas que conformaron la muestra (de tipo censal), excepto por la recusa de tres enfermeras en el Hospital A. Entre los criterios de inclusión se consideró: tiempo de servicio en el área mayor de 12 meses y participación voluntaria; siendo excluidas las enfermas pasantes y aquellas con función administrativa.

Para medir los factores estresores se utilizó el cuestionario "Respuesta Emocional al Estrés Laboral", de Joanna Fornés Vives, citado por Coronado; ${ }^{20}$ instrumento que contiene 36 ítems distribuidos en tres dimensiones: Presión y exigencia (12 ítems), Organizativos y relaciones humanas (12 ítems) y Ambientales (12 ítems), cuyas respuestas son de opción múltiple calificadas como: "1 $1=\mathrm{Si}$, es poco estresante", "2 =Sí, es medianamente estresante" y " 3 =Sí, es altamente estresante". La confiabilidad del instrumento mostró valores calificados como "aceptablesbuenos" a nivel general $(\alpha=0,895)$ al igual que en cada dimensión (Presión y Exigencia=0,689,
Organizativos y Relaciones Humanas $=0,747$ y Ambientales= 0,793).

El segundo instrumento fue el Maslach Burnout Inventory (MBI), creado por Cristina Maslach y Jackson en 1981, traducido al castellano y valido por Gil-Monte y Peiró en $1996 .^{21}$ Consta de 22 ítems distribuidos en tres dimensiones: Realización personal (8 ítems), Agotamiento emocional (9 ítems) y Despersonalización (5 ítems); los cuales son evaluados mediante una escala de tipo Likert con 07 opciones de respuestas, considerando valores de cero (0) a seis (6); cada dimensión se clasifica en nivel bajo, medio y alto.

Los datos fueron recolectados por las autoras en agosto del 2014. Inicialmente se cumplió con los trámites administrativos en los hospitales; concluida esta fase, se realizó una entrevista con los jefes de enfermería de la UCI en cada área de estudio dando a conocer el objetivo del estudio. Para el llenado de los instrumentos, en uno de los hospitales se contactó con cada enfermera al culminar el turno laboral, explicándole el propósito del estudio y los procedimientos, además de absolver dudas y una vez convenida su participación firmaban la Hoja de Consentimiento Informado (HCI), luego procedían a responder el cuestionario. En el segundo hospital, este proceso se realizó de forma grupal al inicio del turno, entregando el instrumento y la HCI para ser llenados en horario libre. Las investigadoras retornaban al día siguiente para recibir los cuestionarios.

Para el análisis de datos se procedió a asignar códigos a cada una de las respuestas, siendo digitados en el programa de Microsoft Office Excel 2013 y verificado la calidad de los datos. Luego se transportaron al paquete estadístico SPSS v.21.0 y procesados mediante la estadística descriptiva aplicando frecuencias, promedio $\mathrm{y}$ desviación estándar. El análisis del síndrome de Bornout se realizó utilizando la escala psicométrica propuesta por Maslach y Jackson en 1986, acorde a lo descrito en el estudio de Gamonal et al. ${ }^{22}$ y, el de Respuesta emocional al estrés laboral fue categorizado aplicando la Escala de Staninos quedando constituida con los siguientes puntajes: en general: alto $=84-108$, medio $=70-83$ y bajo $=36-69$; por dimensiones, Presión y Exigencia: alto $=29-36$, medio $=23-28$ y bajo $=12-22$, Organizativos y Relaciones Humanas: alto $=29-36$, medio $=23-28$ y bajo $=12$ 22 y, Ambientales: alto $=30-36$, medio $=22-29$ y 
bajo=12-21. El análisis bivariado se hizo con la prueba de chi $^{2}$ de Pearson mediante la elaboración de tablas de contingencia que pudieran ser analizadas para establecer la independencia estadística de las variables, considerando un valor significativo de $p=0,05$.

El proyecto del estudio fue evaluado y aprobado por la Oficina de Capacitación, Investigación y Docencia de cada hospital (Carta No. 1595-OCID-G-RAA-ESSALUD-2015); habiéndose respetado los principios éticos aplicados en la investigación con seres humanos: autonomía en la cual cada enfermera tomó la decisión de participar libremente sin coacción del investigador; beneficencia actuando en todo momento a favor de cada participante y porque los resultados contribuirán al cuidado de la salud del profesional; no maleficencia respetando siempre su bienestar físico y emocional; finalmente, de justicia porque todos recibieron un trato equitativo y no se discriminó a ningún participante.

\section{RESULTADOS}

Las enfermeras de UCI $(\mathrm{N}=114)$, en su mayoría tenían edades entre 31-35 años (39\%/44), estando el $66 \%$ (75) casada, con régimen laboral contratadas $(66 \% / 75)$ y una experiencia laboral de 5-9 años (61\%/69), destacando que el 82\% (94) registró tener un trabajo extra.

En el gráfico 1, las enfermeras registran presencia de los factores estresores en nivel medio. Situación que se repite en el análisis por dimensiones, aunque en las dimensiones "organización y relaciones humanas" (24\%/27) y "ambientales" $(22 \% / 25)$ las enfermeras se clasificaron en nivel alto, respectivamente (gráfico 2).

Gráfico 1. Clasificación de los factores estresores de UCI según nivel. Hospital de EsSalud Nivel III2 Lima Metropolitana. Agosto 2014.

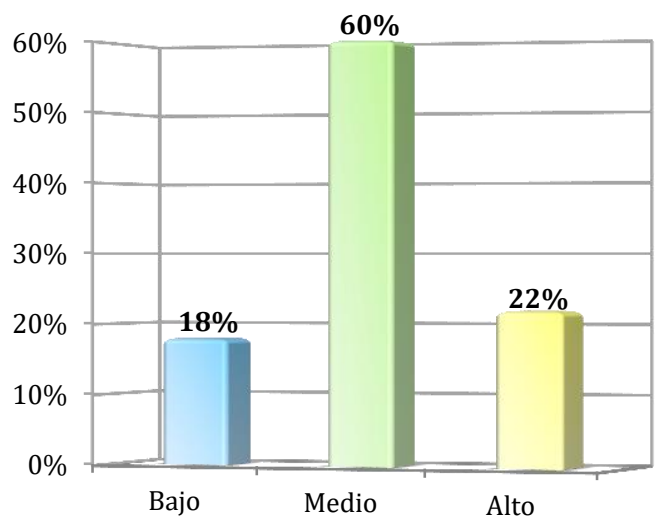

Gráfico 2. Distribución de los factores estresores en enfermeras de UCI por dimensión según nivel. Hospital de EsSalud nivel III-2 Lima Metropolitana. Agosto 2014.

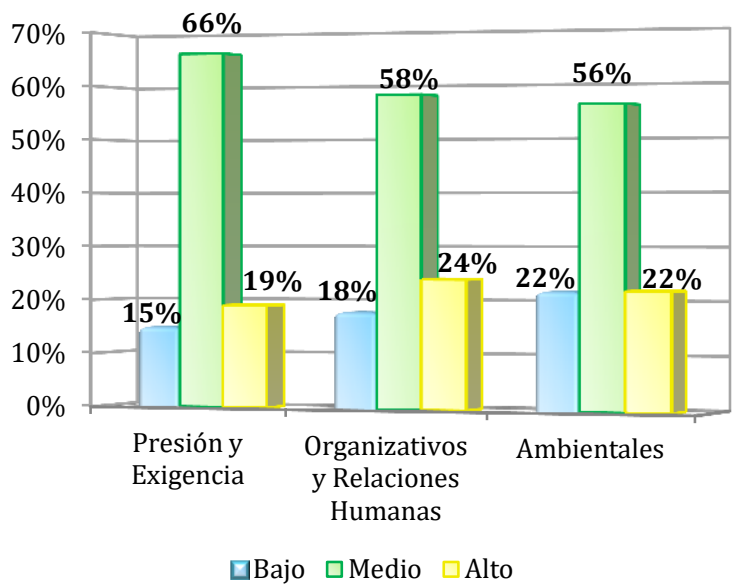

Gráfico 3: Clasificación del síndrome de Burnout en enfermeras de UCI según nivel. Hospital de EsSalud nivel III-2 Lima Metropolitana. Agosto 2014.

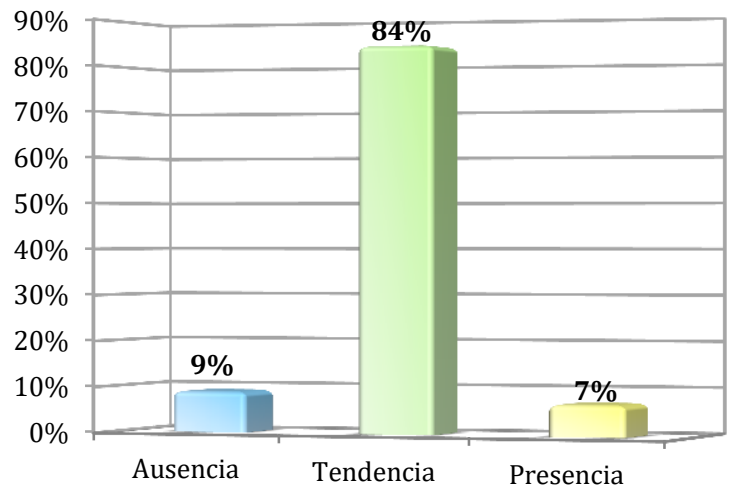

Gráfico 4: Distribución del síndrome de Burnout en enfermeras de UCI por dimensiones según nivel. Hospital de EsSalud nivel III-2 Lima Metropolitana. Agosto 2014

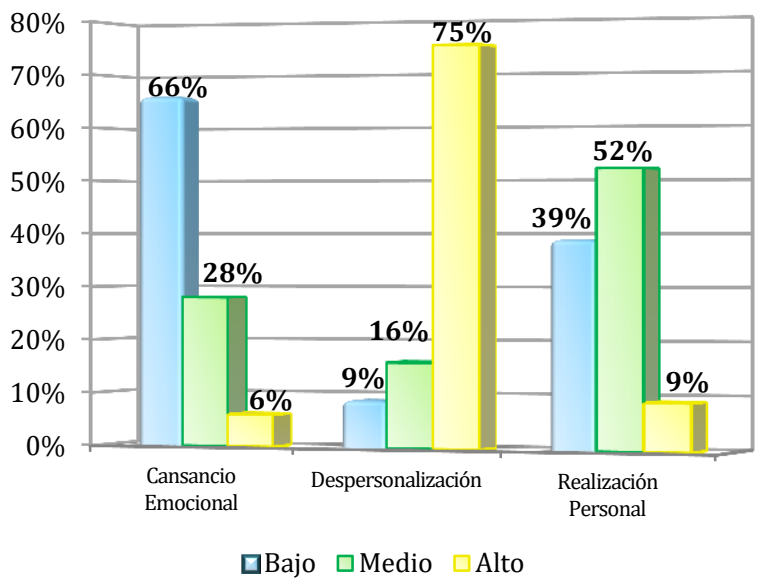


Tabla 1: Relación entre factores estresores y síndrome de Burnout en enfermeras de UCI. Hospital de EsSalud Nivel III-2 Lima Metropolitana. Agosto 2014

\begin{tabular}{|c|c|c|c|c|c|c|c|c|c|}
\hline \multirow{3}{*}{\multicolumn{2}{|c|}{$\begin{array}{c}\text { Factores } \\
\text { estresores }\end{array}$}} & \multicolumn{6}{|c|}{ Síndrome de Burnout } & \multirow{3}{*}{$\mathrm{Chi}^{2}$} & \multirow{3}{*}{$p$} \\
\hline & & \multicolumn{2}{|c|}{ Presencia } & \multicolumn{2}{|c|}{ Tendencia } & \multicolumn{2}{|c|}{ Ausencia } & & \\
\hline & & $\mathrm{n}$ & $\%$ & $\mathrm{n}$ & $\%$ & $\mathrm{n}$ & $\%$ & & \\
\hline \multirow{3}{*}{ 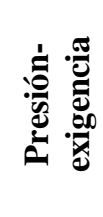 } & Alto & 6 & 5 & 13 & 11 & 3 & 3 & & \\
\hline & Medio & 2 & 2 & 70 & 61 & 3 & 3 & 18,14 & 0,0012 \\
\hline & Bajo & 2 & 2 & 13 & 11 & 2 & 2 & & \\
\hline \multirow{3}{*}{ 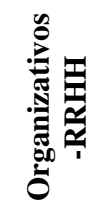 } & Alto & 5 & 4 & 17 & 15 & 5 & 4 & & \\
\hline & Medio & 3 & 3 & 61 & 54 & 2 & 2 & 13,88 & 0,0076 \\
\hline & Bajo & 2 & 2 & 18 & 16 & 1 & 1 & & \\
\hline \multirow{3}{*}{ 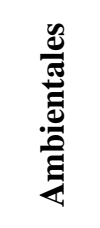 } & Alto & 6 & 5 & 15 & 13 & 4 & 4 & & \\
\hline & Medio & 3 & 3 & 58 & 51 & 3 & 3 & 15,49 & 0,0037 \\
\hline & Bajo & 1 & 1 & 23 & 20 & 1 & 1 & & \\
\hline
\end{tabular}

$\mathrm{gl}=4$ Valor Crítico Chi cuadrado $=5,99$

En el gráfico 3, la mayoría de enfermeras registró tendencia a padecer el síndrome de Burnout $y$ un significativo porcentaje ya lo padecía. Consecuentemente, en el gráfico 4, destaca la dimensión "despersonalización” con nivel alto, mientras que en "realización personal" el nivel medio y bajo.

Al correlacionar las variables se encontró relación significativa $(p<0,05)$ en todos los factores estresores con el síndrome de Bornout (tabla 1), destacándose que el $84 \%$ del personal de enfermería tenía una tendencia al síndrome de Burnout y $7 \%$ ya estaba quemado.

\section{DISCUSIÓN}

La cantidad de horas diarias que el profesional de enfermería pasa junto al paciente, así como el hecho de ser la persona que habitualmente hace de intermediario ante el paciente, el médico y sus familiares son algunas de las razones que hace más vulnerable a este colectivo frente al síndrome de Burnout. ${ }^{23}$ Hecho que se agudiza cuando el profesional labora en áreas críticas como una UCI, que por su propia naturaleza resulta un ambiente estresante, dónde pacientes y familiares están sumidos en un entorno de sufrimiento e incertidumbre, al mismo tiempo que exigen del profesional alta competitividad para el cuidado y manejo de la tecnología avanzada; resultando en alta presión laboral y psicoemocional para la enfermera, con graves repercusiones en su desempeño y estado de salud en general.

La OMS viene promoviendo desde los noventa entornos laborales saludables, especialmente para los profesionales de la salud; en su definición no solo hace hincapié a la ausencia de la enfermedad y a un ambiente físico de trabajo seguro y sin riesgos (multifactoriales), sino que también incorpora a los hábitos de salud (estilos de vida) y los factores psicosociales (organización del trabajo y cultura de trabajo), que se complementan con la comunidad, de modo tal, que promueva la salud integral del empleado. ${ }^{2}$ Identificándose que las acciones de cuidado a los trabajadores deben partir desde la prevención primaria, es decir, evitar que sucedan accidentes o enfermedades de cualquier naturaleza y cuando esto ocurra, garantizar una pronta recuperación, incluso dentro de los ambientes laborales.

Los resultados del estudio muestran que las enfermeras se encontraban en la edad adulta, en su mayoría constituyendo una familia y ejecutando de forma paralela un segundo empleo. Situación que aumenta su vulnerabilidad para desarrollar episodios de estrés, especialmente porque se multiplica el sobreesfuerzo físico y emocional, 
dado que aparte de trabajar 36 horas semanales en el hospital, el $82 \%$ desempeñaba doble empleo; accionar que puede ser consecuencia de la modalidad de contrato laboral. En el estudio predominó el contrato temporal, que suprime ciertos derechos laborales a larga escala, entre ellos una remuneración digna y suficiente para alcanzar una buena vida, debiendo estar a la expectativa de la renovación de su contrato; obligándolas a contar con otras alternativas laborales en detrimento de su salud y bienestar personal y familiar, pues las escasas horas de descanso no terminan de reponer el desgaste físico que implica atender a pacientes críticos y sus familias demandantes.

Sabido es que en los últimos años, con las consecuentes reformas desarrolladas en el sector salud, tendientes en su gran mayoría a la disminución del gasto público, a la descentralización y, a la separación entre la financiación y la prestación de servicios; la situación de los recursos humanos ha sido descuidada, aumentando en muchos casos la precariedad de las condiciones de trabajo, las largas jornadas y la vulnerabilidad de los contratos laborales. $^{15}$

Al respecto, la OPS afirma que existe una creciente, aunque poca evidencia, de que las condiciones de empleo y de trabajo se han deteriorado en los últimos años en todo el mundo; siendo necesario plantear y replantear políticas e instituciones sostenibles tendientes a mejorar dichas condiciones y asegurar una mejor contribución del personal a los resultados sanitarios de los sistemas de salud. ${ }^{15}$ Entendiendo que las condiciones de trabajo y salud tienen un fuerte impacto en la prestación de los servicios de salud y, ciertamente, en la salud de la población.

De acuerdo con esta institución, una situación muy generalizada en América Latina es el pluriempleo en los trabajadores de salud, porcentaje que es elevado en el personal médico, originado principalmente por la oferta de trabajo a tiempo parcial, las bajas remuneraciones que se ofrecen y el mercado "dual" de trabajo entre lo público y lo privado. En el Perú, el 29\% de profesionales enfermeros entre 31-40 años normalmente tiene más de un empleo y, cerca del $90 \%$ trabaja entre $36-48$ horas a más semanalmente. $^{15}$

Un tipo de contrato laboral predominante en el país es el denominado Contratación
Administrativa de Servicios (CAS) y la mayoría de profesionales de enfermería trabaja bajo esta modalidad, con alta restricción de los beneficios laborales versus una remuneración más elevada, que puede ser atractiva para los profesionales noveles, pero que en realidad no proporciona satisfacción al trabajador; muy por el contrario, genera inequidad entre ellos e incluso afecta las relaciones interpersonales. Hecho que ha suscitado interés en los analistas laborales que han propuesto que se mejoren las condiciones de las contrataciones, incluyendo aspectos relacionados con el mérito.

Las excesivas horas laborales, definitivamente tienen impacto en la salud. El 7\% de enfermeras de la UCI esta quemada y $84 \%$ tiene tendencia al síndrome de Burnout (gráfico 3), demostrando que el personal estaba agotado emocionalmente, con sentimientos de despersonalización y mediana realización personal. Solo el $9 \%$ se sentía realizada profesionalmente y $75 \%$ presentaba signos y síntomas de despersonalización (gráfico 4). Es decir, convivía con sentimientos de negatividad y reflejaba actitud de cinismo en su quehacer, siendo posiblemente identificadas por el paciente como enfermeras deshumanizadas.

Cabe señalar que el Burnout provoca una disminución de la habilidad en el desempeño profesional y la aparición de diferentes problemas de salud. Poncet et al. ${ }^{9}$ refieren que las enfermeras con síndrome de Burnout en su forma grave (quemada) presentaron entre otros problemas, interrupción del sueño, insomnio, irritabilidad, problemas con la alimentación, facilidad para el olvido, desórdenes de la libido y síntomas depresivos.

Consecuencias que deben tomarse en cuenta, dado que el $7 \%$ de la población en estudio tenía Burnout. Porcentaje significativo, semejante a los obtenidos en el estudio regional organizado por la OPS el 2010 incluyendo Argentina, Brasil, Costa Rica y Perú, donde este último mostró que afecta al $11 \%$ de enfermeras de los hospitales nacionales, aunque fue el más bajo comparado con los otros países.

Un estudio de pequeño porte realizado en España, considerando al profesional enfermero y al técnico de enfermería que labora en UCI, identificó que el 45,6\% tenía tendencia a padecer síndrome de Burnout y 14,7\% estaba quemado, con baja realización personal $(39,9 \%)$, alta 
despersonalización $(23,5 \%)$ y alto cansancio emocional $(19,1 \%)^{8}$

Un segundo estudio multicentrico, de grande porte, realizado en Francia solo con enfermeras de UCI reportó que un $32,8 \%$ de ellas tenía síndrome de Burnout en nivel grave. Estando asociado al síndrome las características personales [como la edad, el factor organizacional (como la habilidad para elegir sus días libres, participación en grupos de investigación en UCI)], calidad de las relaciones en el trabajo (como los conflictos con los pacientes, relación con la enfermera jefe o médico) y factores relacionados con la muerte/finitud de la vida (como el cuidado al paciente moribundo, las veces que tuvieron que decidir adelantar el tratamiento del paciente para mantenerlo vivo en la última semana). ${ }^{9}$

Situaciones que se presentan de forma común en una unidad de cuidado crítico y condicionan fuertemente el desempeño laboral del profesional enfermero, su satisfacción, su crecimiento, su autonomía. En este contexto se hace necesario y urgente contar con el apoyo de personal supervisor o jefe, quien con sus años de experiencia y adecuación a un trabajo de alta y constante presión, puede ser de gran ayuda principalmente para las enfermeras noveles. No obstante, la realidad muestra que las relaciones entre el personal de la UCI, con los pacientes y con los jefes o supervisores no siempre son buenas, constituyéndose esta relación en uno de los factores estresores.

Poncet et al., ${ }^{9}$ basado en la literatura refieren que el clima en el ambiente de trabajo y la carga laboral son determinantes para el síndrome de Burnout. Identificando que existen altos niveles de Burnout en oncologístas, anestesiologistas, médicos que cuidan de pacientes con SIDA y médicos que trabajan en departamentos de emergencia.

Entre los factores estresores en el estudio prevaleció el nivel medio (gráfico 1), destacando en el análisis por dimensiones, los factores "presión y exigencia” y "organizativos y relaciones humanas". Lo que significa una elevada carga laboral e inadecuación a las normas del servicio y posible relaciones deficiente con los colegas y pacientes, muchas veces por no saber cómo manejar las situaciones, entre ellas las situaciones extremas de vida-muerte, escaso apoyo y falta de insumos.
Zambrano $^{24}$ revela que el conflicto con los superiores, la sobrecarga laboral y el contacto con el dolor y la muerte son los principales estresores en una UCI, resaltando que las enfermeras se preocupan por las "consecuencias de sus errores con el enfermo", el "tener un superior incompetente", la "escasez de recursos esenciales" y la "sobrecarga del trabajo". Datos semejantes fueron reportados por Simon et al. ${ }^{25}$ en España.

Llama la atención en el estudio el porcentaje de nivel alto alcanzado por los factores "organizativos y relaciones humanas" y "ambientales" (gráfico 2), que se encuadran con los estresores descritos por los autores arriba citados. Coronado, ${ }^{20}$ citando a Nuñez, describe que los factores "organizativos" son inherentes a la institución y están relacionados con los turnos de trabajo, la sobrecarga laboral, el clima organizacional, los estilos de gerencia, entre otros. A su vez, los factores "ambientales" se refieren a las condiciones físicas externas que pueden alterar el curso normal del desempeño profesional, distorcionando la concentración y tranquilidad que requiere el cumplimiento de su función de manera óptima, tales como el ruido, iluminación, vibraciones, contaminación, temperatura, limitaciones de espacio, disposición de herramientas/instrumental, entre otros.

En general, las enfermeras se sienten muy preocupadas por el paciente, con su sufrimiento, impotencia de no poder ayudarlo más, la eficiencia en el cuidado que realiza y al mismo tiempo le preocupa causar más dolor, la incompetencia de no poder ayudar emocionalmente a la familia del paciente, entre otros aspectos que le son propios del trabajo. ${ }^{25}$ Donde además tienen que lidiar con las carencias de insumos y equipos, falta de apoyo de los supervisores y de ayuda ante lo desconocido, ambientes de trabajo conflictivos. ${ }^{26}$

Actualmente, los factores organizativos y de relaciones humanas en el espacio laboral se identifican como factores psicosociales y constituyen una preocupación demandante para la OMS. En concordancia con Gil-Monte, ${ }^{27}$ estos factores constituyen las condiciones de una situación laboral directamente relacionadas con la organización del trabajo, con el contenido del puesto, con la realización de la tarea, inclusive con el entorno; los cuáles tienen la capacidad de afectar el desarrollo del trabajo y la salud de los trabajadores. 
Ambos factores están inmersos en la complejidad de la interacción humana, la multicuturalidad, valores personales, normatividad, satisfacción y realización humana, intereses del empleador, patrones de la cultura organizacional, eficiencia en el desarrollo del cuidado, entre otros; conllevando finalmente a un desgaste físico y emocional.

En tal sentido, los factores psicosociales son reconocidos como una de las principales causas de enfermedades y de accidentes laborales. GilMonte, ${ }^{27}$ basado en el análisis de diferentes estudios, resalta que los factores psicosociales son responsables del alto grado de estrés entre los trabajadores con graves repercusiones en la salud y el trabajo, lo que supone un coste económico y social importante; debido al ausentismo laboral, accidentes laborales, sobrecarga laboral; siendo una consecuencia directa el síndrome de quemarse o Burnout, porcentajes que oscilan entre 2,4-30\% en diversos países de Europa y EUA. Interín, en Latino América los porcentajes van de 11-55\%, siendo que el Perú presenta la cifra más baja $(11 \%)$.

Los resultados del estudio muestran que existe relación entre el síndrome de Burnout y los factores estresores en las enfermeras de la UCI (tabla 2), es decir que los límites de tolerabilidad al estrés ya fueron superados. Mostrando que los factores están presentes de manera importante y que es necesario intervenir con acciones que protejan al personal de salud, promoviendo entornos laborales saludables, acorde con las necesidades de cada contexto, desde los cambios en las directrices políticas de las instituciones sobre los recursos humanos, hasta la implementación de mejoras en los espacios físicos del servicio, además de promover una mayor comunicación entre los trabajadores y directivos, flexibilización de las normas internas del servicio y acompañamiento del equilibro familiar, en aras de construir a mediano y largo plazo, un entorno laboral saludable.

De acuerdo con la OMS, ${ }^{2}$ un entorno laboral saludable se caracteriza porque los trabajadores y jefes colaboran en un proceso de mejora continua para promover y proteger la salud, la seguridad y el bienestar de los trabajadores y la sustentabilidad del ambiente de trabajo.

Aquí, es necesario reconocer la responsabilidad del empleador, quien debe proporcionar las condiciones óptimas para la seguridad del trabajador, no apenas en cuestión de infraestructura y equipamiento de las unidades criticas como el área de cuidado intensivo, sino generando propuestas que aseguren un factor psicosocial que aporte equilibrio emocional, afrontamiento oportuno frente a las situaciones adversas provocadas en ocasiones por las diferencias en las relaciones interpersonales, esto logrará un mayor aporte para la salud del profesional enfermero y los demás miembros del equipo de salud.

La OPS, ${ }^{2}$ citando el estudio de Burton et al., refiere que a medida que los factores de riesgo para la salud aumentan, la productividad disminuye. Es decir, que disminuye la calidad del cuidado que ofrece, con insatisfacción del paciente o tal vez, con complicaciones que a la larga afecta al sistema de salud, por los largos períodos de hospitalización que esto genera, ofertando una menor capacidad resolutiva y altos costos directos e indirectos.

Poncet et al., ${ }^{9}$ sugieren trabajar la prevención de conflictos, participación en grupos de investigación, mejorar la gestión del cuidado al final de la vida. La gran responsabilidad del profesional de enfermería es proporcionar al paciente y familia, la comprensión de un hecho que va unido a la vida desde el nacimiento, como lo es la muerte.

Gestionar el cuidado también implica salvaguardar la vida y la salud de los profesionales de enfermería, para ello se requiere de una investigación que identifique la problemática actual de la profesión y pueda estructurar las opciones más acordes para formalizar la salud laboral desde el propio trabajador.

En conclusión, el estudio mostró presencia en mediano nivel de los factores estresores en la UCI y que el $7 \%$ de los profesionales estaba con sintomatología de Burnout (quemado), además que el $85 \%$ está en camino a desencadenarlo. Resultados que son preocupantes y que ameritan ser abordada por los directivos y gestores de las instituciones involucradas, por las graves consecuencias que desencadena y porque es prioritario y mandatorio establecer espacios laborales adecuados y seguros a los trabajadores, conforme a las regulaciones y normatividad internacional.

Por el diseño del estudio, los resultados solo son aplicados a los enfermeros de los hospitales 
participantes, sin embargo, constituyen un referente para seguir investigando esta temática en

\section{Correspondencia:}

Maria del Rosario Menor Segura

Correo electrónico: menor_segura@hotmail.com

\section{REFERENCIAS BIBLIOGRÁFICAS}

1. World Health Organization (WHO) [Editorial]. Br Med J [serie de internet]. 2000 [citado 01 de dic 2012]; 320:237-43. Disponible en: http://www.who.int/mediacentre/factsheets/fs3 $\underline{89 / \mathrm{es} /}$

2. World Health Organization. Entornos Laborales Saludables: Fundamentos y Modelos de la OMS Contextualización, Prácticas y Literatura de Apoyo. [internet]. Ginebra: OMS; 2010 [citado 25 de abr 2013]. p.144. Disponible en: http://www.who.int/occupational_health/evelyn hwp spanish.pdf

3. Health and Safety Executive (HSE). Health and safety in human health and social care in Great Bretain, 2014/15 [internet]. Great Bretain: HSE; 2008. [citado 20 de jun 2013]. p.21. Disponible en: http://www.hse.gov.uk/statistics/industry/healt hservices/health.pdf

4. Roel VJM, Maqueda BJ. Ensayo de campo de un procedimiento de investigación de casos de enfermedades de origen laboral. Med Segur Trab [serie de internet]. Abr-jun 2013 [citado 4 de jun 2015], 59(231): 227-234. Disponible en: http://scielo.isciii.es/pdf/mesetra/v59n231/origi nal2.pdf

5. Organización Internacional del Trabajo. Prevención de enfermedades profesionales. Safework. [Internet]. Ginebra: OIT; mar 2013 [citado 10 de ene 2014]. p.13. Disponible en: http://www.ilo.org/wcmsp5/groups/public/--ed_norm/--relconf/documents/meetingdocument/wcms_20 4788.pdf

6. Díaz PC. Síndrome de Quemazón (Burnout) en el personal de salud. Caguas-Puerto Rico: EDIC College; 2012 [citado 29 de abr 2013]. p.16. Disponible en: http://ediccollege.edu/upload/pdf/EducacionCo otros hospitales del país e implementar las innovaciones necesarias. $\underline{\text { ntinuaPDF/Sindrome de Quemazon_en_el_Pe }}$ rsonal_de_Salud.pdf

7. Organización Colegial de Enfermería. Sala de Prensa [internet]. España: Consejo General de Colegios Oficial de Enfermería de España; c2014-2016. Sala de Prensa. El 96\% de enfermeras se ha sentido estresada en alguna ocasión en su lugar de trabajo; 2014 [citado 15 de ene 2015]; [aproximadamente 2 aludas]. Disponible en: http://www.consejogeneralenfermeria.org/inde x.php/sala-de-prensa/noticias/item/5405-el-96de-los-enfermeros-se-ha-sentido-estresado-enalguna-ocasi\%C3\%B3n-en-su-lugar-detrabajo\#.VhvsJux_Okq

8. Sánchez AP, Sierra OVM. Síndrome de Burnout en el personal de enfermería en UVI. Enferm glob [serie de internet]. Ene 2014 [citado 3 de ene 2015]; 13(33):252-7. Disponible en: http://revistas.um.es/eglobal/article/view/17681 $\underline{1 / 157191}$

9. Poncet MC, Toullic P, L Papazian, KentishBarnes N, Timsit JF, Pochard F, et al. Burnout syndrome in critical care nursing staff. Am J Respir Crit Care Med [serie de internet]. Apr 2007 1[citado 27 de set 2013]; 175(7): 698$704 . \quad$ Disponible en: http://www.atsjournals.org/doi/pdf/10.1164/rcc m.200606-8060C

10. Navarro FMA. Prevalencia del Síndrome de Burnout en los Profesionales de Enfermería del Hospital General Obispo Polanco en Teruel [tesis de pregrado]. Zaragoza: Escuela Universitaria de Enfermería [internet]; 2014 [citado 15 dic de 2015]. Disponible en: https://zaguan.unizar.es/record/16253/files/TA Z-PFC-2014-489.pdf

11. Arias GP, Castro LM. Prevalencia del Síndrome de Burnout y Factores Sociodemográficos y Laborales Asociados e Enfermería (as) Profesionales del Hospital 
nacional de Niños durante el año 2012 [tesis de postgrado]. San José: Universidad de Costa Rica [internet]; 2012 [citado 21 de jun 2013]. Disponible en: http://www.binasss.sa.cr/bibliotecas/bhp/textos /tesis38.pdf

12. Tenesaca MJA, Uzhca BMA, Valarezo LDC. Factores de Riesgo asociados al Síndrome de Burnout en las Enfermeras Profesionales del Hospital "Homero Castanier Crespo" Azogues2011 [tesis de pregrado]. Loja: Universidad de Cuenca [internet]; 2012 [citado 21 jun de 2013]. Disponible en: http://dspace.ucuenca.edu.ec/bitstream/123456 $\underline{789 / 3667 / 1 / \text { ENF86pdf }}$

13. Comportamiento del Síndrome de Burnout en el personal de enfermería que labora en los servicios de urgencia hospitalario [internet]. s.d.: Revista Electrónica de Portales Médicos. c1999-2015 [citado 2 de jun 2013]. Disponible en:

http://www.portalesmedicos.com/publicaciones /articles/4461/2/Investigacion.-

Comportamiento-del-Sindrome-de-Burnout-enel-personal-de-Enfermeria-que-labora-en-losservicios-de-urgencia-hospitalaria

14. Lopes GFJ, Ferraz BER. Estrés de los Enfermeros de UCI en Brasil. Rev Enferm Glob [serie de internet]. Abr 2011 [citado 20 de jul 2014]; 10(2): 1-9. Disponible en: http://scielo.isciii.es/pdf/eg/v10n22/clinica3.pd $\underline{f}$

15. Organización Panamericana de la Salud. Estudio comparativo de las condiciones de trabajo y salud de los trabajadores de la salud en: Argentina, Brasil, Costa Rica y Perú [internet]. Washington: OPS; 2012 [citado 7 de may 2013].p.142. Disponible en: http://www.renastonline.org/sites/default/files/a rquivos/recursos/HSS-

Cond Trab_RHS2012.pdf

16. Ayala CE. Síndrome de Burnout en el personal de enfermería de los servicios críticos del Hospital Central de la Fuerza Aérea del Perú en el año 2011 [tesis de postgrado]. Lima-Perú: Universidad Nacional Mayor de San Marcos [internet]. 2013 [citado 13 ago 2014]. Disponible en: http://ateneo.unmsm.edu.pe/ateneo/bitstream/1 23456789/2684/1/Ayala_Cardenas_Elizabeth 2013.pdf
17. Mera ODA, More DEN. dimensiones del síndrome de burnout entre profesionales de enfermería de áreas críticas de un hospital MINSA y EsSalud de Chicalyo-2013. [tesis de pregrado]. Chiclayo: Universidad Católica Santo Toribio De Mogrovejo [internet]. 2013 [citado 14 de may 2015]. Disponible en: http://tesis.usat.edu.pe/jspui/bitstream/1234567 89/283/1/TL_MeraOlivaresDiego_MoreDuran Erika.pdf

18. El Comercio [internet]. Lima-Perú: El Comercio, c.s.f.-2016. El 78\% de los trabajadores peruanos confesó haber sufrido de estrés laboral; dic 16 de 2009 [citado 16 de ene 2013]; [aproximadamente una laudas]. Disponible en: http://elcomercio.pe/economia/negocios/78trabajadores-peruanos-confeso-sufrido-estreslaboral-noticia-382259

19. Juárez AG. Entrevista con Christina Maslach: Reflexiones sobre el Síndrome de Burnout. Liberabit [internet]. 2014 [citado 16 de oct 2015]; 20(2):199-208. Disponible en: http://www.scielo.org.pe/pdf/liber/v20n2/a01v $\underline{20 n 2 . p d f}$

20. Coronado LLK. Factores Laborales y Nivel de Estrés Laboral en Enfermeros de los Servicios de Áreas Críticas y Medicina del Hospital Nacional Daniel A. Carrión. 2006 [tesis de pregrado]. Lima-Perú: Universidad Nacional Mayor de San Marcos [internet]. 2010 [citado 24 de oct 2013]. Disponible en: http://cybertesis.unmsm.edu.pe/bitstream/cyber tesis/1011/1/Coronado_11.pdf

21. Gil-monte PR, Peiró JM. Validez factorial del Maslach Burnout Inventory en una muestra Multiocupacional. Rev Psicothema [internet]. 1996 [citado 10 de dic 2015]; 11(3): 679-689. Disponible en: http://www.unioviedo.es/reunido/index.php/PS $\underline{\text { T/article/view/7551/7415 }}$

22. Gamonal MYL, García VCM, Silva MZI. Síndrome de burnout en El profesional de enfermería que labora en áreas críticas. Rev enferm Herediana [serie de internet]. Ene-jun 2008 [citado 10 de nov 2013]; 1(1):33-9. Disponible en: http://www.upch.edu.pe/faenf/images/pdf/Revi stas/2008/enero/v1n1ao5.pdf 
23. Gómez SC, Álamo SC, Amador BM, Ceacero MF, Mayor PA, Muñoz GA et al. Estudio de seguimiento del desgaste profesional en relación con factores organizativos en el personal de enfermería de medicina interna. Med Segur Trab [serie de internet]. Abr-jun 2009 [citado 10 de agos 2015]; 55(215): 52-62. Disponible en: http://scielo.isciii.es/pdf/mesetra/v55n215/origi $\underline{\text { nal3.pdf }}$

24. Zambrano PGE. Estresores en las unidades de cuidado intensivo. Aquichan [serie de internet]. Oct 2006 [citado 15 de dic 2015]; 6(1): 156-69. Disponible en: http://www.redalyc.org/articulo.oa?id=741601 $\underline{16}$

25. Simon GMJ, Blesa MAL, Bermejo PC, Calvo GMA, Enterría PGC. Estresores laborales y satisfacción en la enfermería de una unidad de críticos. Enferm Intensiva [serie de internet]. 2005 [citado 15 de dic 2015]; 16(1): 3-14. Disponible en: http://www.elsevier.es/esrevista-enfermeria-intensiva-142-articuloestresores-laborales-satisfaccion-enfermeriauna-13072134

26. Andrade ECA. Factores que condicionan estrest en el personal de enfermería [tesis de postgrado]. Querétaro-México: Universidad Autónoma de Querétaro [internet]. 2011 [citado 15 de dic 2015]. Disponible en: http://ri.uaq.mx/bitstream/123456789/1029/1/R I000545.pdf

27. Gil.Monte PR. Algunas razones para considerar los riesgos psicosociales en el trabajo y sus consecuencias en la salud pública. Rev Esp Salud Pública [serie de internet]. 2009 [citado 10 de oct 2015]; 83: 169-73. Disponible en: http://scielo.isciii.es/pdf/resp/v83n2/editorial3. pdf 